\title{
ON A ONE-DIMENSIONAL $\alpha$-PATCH MODEL WITH NONLOCAL DRIFT AND FRACTIONAL DISSIPATION
}

\author{
HONGJIE DONG AND DONG LI
}

\begin{abstract}
We consider a one-dimensional nonlocal nonlinear equation of the form $\partial_{t} u=\left(\Lambda^{-\alpha} u\right) \partial_{x} u-\nu \Lambda^{\beta} u$, where $\Lambda=\left(-\partial_{x x}\right)^{\frac{1}{2}}$ is the fractional Laplacian and $\nu \geq 0$ is the viscosity coefficient. We primarily consider the regime $0<$ $\alpha<1$ and $0 \leq \beta \leq 2$ for which the model has nonlocal drift, fractional dissipation, and captures essential features of the $2 \mathrm{D} \alpha$-patch models. In the critical and subcritical range $1-\alpha \leq \beta \leq 2$, we prove global wellposedness for arbitrarily large initial data in Sobolev spaces. In the full supercritical range $0 \leq \beta<1-\alpha$, we prove formation of singularities in finite time for a class of smooth initial data. Our proof is based on a novel nonlocal weighted inequality which can be of independent interest.
\end{abstract}

\section{INTRODUCTION}

We consider the following nonlocal nonlinear transport equation of Burgers' type:

$$
\partial_{t} u=\left(\Lambda^{-\alpha} u\right) \partial_{x} u-\nu \Lambda^{\beta} u,
$$

where $\Lambda=\left(-\partial_{x x}\right)^{\frac{1}{2}}$ is the fractional Laplacian, $\nu \geq 0$ is the viscosity coefficient, $0<\alpha<1$, and $0 \leq \beta \leq 2$. In the inviscid case, this equation can be viewed as a $1 \mathrm{D}$ analogy of the $2 \mathrm{D} \alpha$-patch problem

$$
\partial_{t} \theta+u \cdot \nabla \theta=0, \quad u=\left(-\partial_{x_{2}}, \partial_{x_{1}}\right)(-\Delta)^{-(1+\alpha) / 2} \theta,
$$

which represents an interpolation between the 2D Euler and quasi-geostrophic equations; see [4,9,14 for some discussions.

Equation (1.1) becomes more singular as $\alpha$ decreases. It reduces to the inviscid Burgers' equation when $\alpha=\nu=0$, in which case it is well known that solutions may develop gradient blowup in finite time. When $\alpha=0$ and $\beta \in(0,2]$, (1.1) becomes the so-called fractal Burgers' equation, which is perhaps one of the simplest nonlinear equations with nonlocal terms. The fractal Burgers' equation was studied in detail recently; see, for instance, $[1,2,5,13,16,20$. It is known that in the supercritical dissipative case $\beta \in(0,1)$, with very generic initial data the equation is locally well-posed and its solution may develop gradient blowup in finite time. In the critical and subcritical dissipative case $\beta \in[1,2]$, the equation is globally wellposed with arbitrary initial data in suitable Sobolev spaces. Another borderline situation is when $\alpha=1$ and $\nu=0$, in which case the equation is globally wellposed; see 21] for a proof in the periodic boundary condition case. As a matter of

Received by the editors July 18, 2012.

2010 Mathematics Subject Classification. Primary 35Q53, 35B44, 35B65. 
fact, in this case in the same spirit as the Beale-Kato-Majda criterion the solution is regular up to time $T$ as long as

$$
\int_{0}^{T}\|u(t, \cdot)\|_{L^{\infty}} d t<\infty
$$

This holds for any $T$ since (1.1) is a transport equation and $\|u(t, \cdot)\|_{L^{\infty}}$ is nonincreasing.

In this paper, we primarily consider the regime $0<\alpha<1$ and $0 \leq \beta \leq 2$ for which the model has nonlocal drift, fractional dissipation, and captures essential features of the 2D $\alpha$-patch models. We are interested in proving either the global regularity or finite time blowup for (1.1). In the general case, the corresponding regularity criterion turns out to be

$$
\int_{0}^{T}\left\|\partial_{x} \Lambda^{-\alpha} u(t, \cdot)\right\|_{\infty} d t<\infty \quad \text { or } \quad \int_{0}^{T}\left\|\Lambda^{1-\alpha} u(t, \cdot)\right\|_{\infty} d t<\infty
$$

which are not straightforward to verify. See Theorem 2.2 and Remark 2.5,

Let us describe the main results of the paper. In the critical and subcritical range $1-\alpha \leq \beta \leq 2$, we obtain global wellposedness for arbitrarily large initial data in suitable Sobolev spaces. In the full supercritical range $0 \leq \beta<1-\alpha$, we prove formation of singularities in finite time for a family of smooth initial data. These results are in the same spirit as the results in [13,16, for the fractal Burgers' equation.

We state our main results of the paper more precisely in the following two theorems.

Theorem 1.1 (Finite time blowup in the supercritical case). For any $0<\alpha<1$, $0 \leq \beta<1-\alpha, 2 \alpha<\delta<2(1-\beta)$ and $\nu \geq 0$, there is a constant $C_{\alpha, \beta, \delta, \nu}>0$ sufficiently large such that the following hold true:

For any smooth initial data $u_{0} \in C_{c}^{\infty}(\mathbb{R})$ which is odd in $x$ and satisfies:

a) $u_{0}(x) \geq 0$, for any $x \geq 0$,

b)

$$
\int_{0}^{\infty} \frac{u_{0}(x)}{x^{\delta-\alpha}} d x \geq C_{\alpha, \beta, \delta, \nu}\left(1+\left\|u_{0}\right\|_{L_{x}^{1}}\right),
$$

the corresponding solution to (1.1) blows up in finite time.

Theorem 1.2 (Global wellposedness in the critical and supercritical cases). Let $0<\alpha<1,1-\alpha \leq \beta \leq 2$, and $\nu>0$. Assume $1<p<\frac{1}{\alpha}$ and set $k_{0}=$ $\max \left\{\frac{\frac{1}{p}-\frac{1}{2}}{\frac{1}{p}-\alpha}, 2\right\}$. Suppose that the initial data $u_{0} \in L^{p} \cap H^{k}$ for some integer $k>k_{0}$. Then there exists a unique solution $u$ to (1.1) in the space $C\left([0, \infty), L^{p} \cap H^{k}\right)$ with $u(0)=u_{0}$. Moreover, $u \in C\left((0, T), L^{p} \cap H^{k^{\prime}}\right)$ for any $k^{\prime} \geq k$.

For the proof of Theorem 1.1, we consider the evolution of a weighted integral of $\Lambda^{-\alpha} u$. The main difficulty is to show that such a quantity satisfies some ordinary differential inequality, and would blow up in finite time under conditions a) and b) above. This then implies the blowup of the solution. Before, this type of approach could be found, for instance, in [3, 17, 8, 12, 18, 19]. We point out that, in these papers, weighted integral of solutions themselves are investigated, which unfortunately does not seem to work in our case. The key new observation in our case is that instead of working with the solution $u$ itself, it is more natural to consider 
a certain weighted integral of the nonlocal quantity $\Lambda^{-\alpha} u$ and establish positive lower bounds expressed in terms of $\Lambda^{-\alpha} u$. More precisely we have the following nonlocal weighted inequality, which could be of independent interest.

Proposition 1.3 (A nonlocal weighted inequality). Let $0<\alpha<1$ and $2 \alpha<\delta<2$. There is a constant $C_{\alpha, \delta}>0$ such that for any odd function $u \in C_{b}^{1}(\mathbb{R}) \cap L_{x}^{1}(\mathbb{R})$, we have

$$
\int_{0}^{\infty} \frac{\Lambda^{-\alpha}\left(\Lambda^{-\alpha} u \cdot \partial_{x} u\right)}{x^{\delta}} d x \geq C_{\alpha, \delta} \int_{0}^{\infty} \frac{\left(\Lambda^{-\alpha} u\right)^{2}}{x^{1+\delta}} d x
$$

Here $C_{b}^{1}(\mathbb{R})=\left\{f \in C^{1}(\mathbb{R}):\|f\|_{L_{x}^{\infty}}+\left\|f^{\prime}\right\|_{L_{x}^{\infty}}<\infty\right\}$.

Remark 1.4. The parity assumption in Proposition 1.3 can probably be removed or relaxed by a more elaborate analysis, but we will not do it here. The nontrivial point of Proposition 1.3 is to guess the correct positive lower bound such as the right-hand side of (1.3). We briefly explain the difficulty as follows. By a simple computation (see e.g. Lemma 3.1), we have for some constant $C_{\alpha, \delta}^{\prime}>0$,

$$
\text { LHS of (1.3) }=C_{\alpha, \delta}^{\prime} \int_{0}^{\infty} \frac{\Lambda^{-\alpha} u \cdot \partial_{x} u}{x^{\delta-\alpha}} d x .
$$

From this and a scaling heuristic, one is led to conjecture the inequality

$$
\int_{0}^{\infty} \frac{\Lambda^{-\alpha} u \cdot \partial_{x} u}{x^{\delta-\alpha}} d x \gtrsim \int_{0}^{\infty} \frac{u^{2}}{x^{1+\delta-2 \alpha}} d x
$$

The inequality (1.4) is close in spirit to the type of inequalities used in [3,, , 8, 12, 18, 19]. However a preliminary calculation shows that the inequality (1.4) is probably false unless some negative terms are added on the right-hand side of (1.4). To circumvent this difficulty we prove (1.3). Note that these two lower bounds are not equivalent, although they obey the same scaling relations.

For the proof of Theorem 1.2 when $\beta<2$ we adapt an idea of the nonlocal maximum principle for a suitably chosen modulus of continuity. This method was first used by Kiselev, Nazarov, and Volberg in [15, where they established the global regularity for the $2 \mathrm{D}$ critical dissipative quasi-geostrophic equations with periodic $C^{\infty}$ data. We also refer the reader to [17] and the references therein for further applications and development of this method. In the case $\beta=2$, this method does not seem to be applicable, and we use a different approach.

The remaining part of the paper is organized as follows. In the next section, we prove the local wellposedness, continuation criteria for (1.1), and Theorem 1.2 In section 3 we present several auxiliary lemmas, which will be used in the proofs of Proposition 1.3 and Theorem 1.1 in the last section.

We close this introduction by setting up some

Notation. For any two quantities $A$ and $B$, we use $A \lesssim B$ (resp. $A \gtrsim B$ ) to denote the inequality $A \leq C B$ (resp. $A \geq C B$ ) for a generic positive constant $C$. The dependence of $C$ on other parameters or constants is usually clear from the context and we will often suppress this dependence. The value of $C$ may change from line to line. For any function $f: \mathbb{R} \rightarrow \mathbb{R}$, we use $\|f\|_{L^{p}}$ or sometimes $\|f\|_{p}$ to denote the usual Lebesgue $L^{p}$ norm of a function for $1 \leq p \leq \infty$.

The fractional Laplacian operator $\Lambda^{s}, s \in \mathbb{R}$, is defined via Fourier transform as

$$
\mathcal{F}\left(|\nabla|^{s} f\right)(\xi)=|\xi|^{s}(\mathcal{F} f)(\xi), \quad \xi \in \mathbb{R} .
$$


The homogeneous Sobolev norm $\dot{H}^{s}$ for any $s \geq 0$ is defined as $\|f\|_{\dot{H}^{s}}=\left\||\nabla|^{s} f\right\|_{2}$, or more explicitly:

$$
\|f\|_{\dot{H}^{s}}=\left(\int_{\mathbb{R}}|\xi|^{2 s}|(\mathcal{F} f)(\xi)|^{2} d \xi\right)^{\frac{1}{2}} .
$$

We will need to use the Littlewood-Paley (LP) frequency projection operators. For simplicity we shall fix the notation on $\mathbb{R}$, but it is straightforward to define everything in $\mathbb{R}^{d}$ for any $d \geq 1$. To fix the notation, let $\phi \in C_{0}^{\infty}(\mathbb{R})$ and satisfy

$$
0 \leq \phi \leq 1, \quad \phi(x)=1 \text { for }|x| \leq 1, \quad \phi(x)=0 \text { for }|x| \geq 2 .
$$

For two real positive numbers $\alpha<\beta$, define the frequency localized projection (LP) operator $P_{\alpha<\cdot<\beta}$ by

$$
P_{\alpha<\cdot<\beta} f=\mathcal{F}^{-1}\left(\left[\phi\left(\beta^{-1} \xi\right)-\phi\left(\alpha^{-1} \xi\right)\right] \mathcal{F}(f)\right) .
$$

Here $\mathcal{F}$ and $\mathcal{F}^{-1}$ denote the Fourier transform and its inverse transform, respectively. Similarly, the operators $P_{<\alpha}$ and $P_{>\beta}$ are defined by

$$
P_{<\beta} f=\mathcal{F}^{-1}\left(\phi\left(\beta^{-1} \xi\right) \mathcal{F}(f)\right)
$$

and

$$
P_{>\alpha} f=\mathcal{F}^{-1}\left(\left[1-\phi\left(\alpha^{-1} \xi\right)\right] \mathcal{F}(f)\right) .
$$

We recall the following Bernstein estimates: for any $1 \leq p \leq q \leq \infty$ and dyadic $N>0$,

$$
\left\|P_{<N} f\right\|_{L^{q}(\mathbb{R})} \lesssim N^{\frac{1}{p}-\frac{1}{q}}\|f\|_{L^{p}(\mathbb{R})}
$$

\section{LOCAL AND GLOBAL REGULARITY}

We first state the following positivity lemma which is a simple variant of Lemma 2.5 in [6]. We include the proof here for the sake of completeness.

Lemma 2.1 (Positivity lemma). Let $F: \mathbb{R} \rightarrow \mathbb{R}$ be a nondecreasing function. Assume $0 \leq \beta \leq 2$. Then for any $\theta: \mathbb{R} \rightarrow \mathbb{R}$ such that $\Lambda^{\beta} \theta$ is well-defined and $F(\theta) \Lambda^{\beta} \theta \in L^{1}(\mathbb{R})$, we have

$$
\int_{-\infty}^{\infty} F(\theta) \Lambda^{\beta} \theta d x \geq 0
$$

In particular, for any $1 \leq p<\infty$,

$$
\int_{-\infty}^{\infty}|\theta|^{p-2} \theta \Lambda^{\beta} \theta d x \geq 0
$$

provided that the integral is well-defined.

Proof. Without loss of generality, we assume $F$ is a smooth function. In the general case one can mollify $F$ and deduce the result by a limiting argument. First consider $\beta=2$. In this case we just integrate by parts and obtain

$$
\int_{-\infty}^{\infty} F(\theta)\left(-\partial_{x x}\right) \theta d x=\int_{-\infty}^{\infty} F^{\prime}(\theta)\left(\partial_{x} \theta\right)^{2} d x \geq 0
$$

since $F$ is nondecreasing. 
Now we assume $0<\beta<2$. Recall that for $0<\beta<1$, we have

$$
\left(\Lambda^{\beta} g\right)(x)=C_{\beta} \lim _{\epsilon \rightarrow 0} \int_{|y-x|>\epsilon} \frac{g(x)-g(y)}{|x-y|^{1+\beta}} d y
$$

for some constant $C_{\beta}>0$.

For $\beta=1$ one can use $\Lambda g=H \partial_{x} g$ ( $H$ is the Hilbert transform) and integration by parts to show that the formula (2.2) still holds. For smooth $g$, an equivalent formula (without $\epsilon$-limit) is given by the expression

$$
(\Lambda g)(x)=C \int_{|y-x|<1} \frac{g(x)-g(y)+g^{\prime}(x)(y-x)}{|y-x|^{2}} d y+C \int_{|y-x|>1} \frac{g(x)-g(y)}{|y-x|^{2}} d y .
$$

Similarly for $1<\beta<2$ one can use $\Lambda^{\beta} g=-\Lambda^{\beta-2} \partial_{x x} g$, a fractional representation of the Riesz potential $\Lambda^{-(2-\beta)}$ and integration by parts (twice) to show that (2.2) also holds in this case. In this case a formula equivalent to (2.2) is given by

$$
\left(\Lambda^{\beta} g\right)(x)=C_{\beta} \int_{\mathbb{R}} \frac{g(x)-g(y)+g^{\prime}(x)(y-x)}{|x-y|^{1+\beta}} d y .
$$

In all cases we shall just use fractional representation of $\Lambda^{\beta}$ as in (2.2). Clearly

$$
\begin{aligned}
& \int_{-\infty}^{\infty} F(\theta) \Lambda^{\beta} \theta d x \\
= & C_{\beta} \int_{\mathbb{R}_{x}^{1}} F(\theta(x)) \lim _{\epsilon \rightarrow 0} \int_{|y-x|>\epsilon} \frac{\theta(x)-\theta(y)}{|x-y|^{1+\beta}} d y d x \\
= & \lim _{\epsilon \rightarrow 0} C_{\beta} \int_{\mathbb{R}_{x}^{1}} F(\theta(x)) \int_{|y-x|>\epsilon} \frac{\theta(x)-\theta(y)}{|x-y|^{1+\beta}} d y d x .
\end{aligned}
$$

Symmetrizing the above integral in $x$ and $y$, we obtain

$$
\begin{aligned}
& \int_{-\infty}^{\infty} F(\theta) \Lambda^{\beta} \theta d x \\
= & \frac{1}{2} C_{\beta} \lim _{\epsilon \rightarrow 0} \int_{\mathbb{R}_{x}^{1}} \int_{|y-x|>\epsilon}(F(\theta(x))-F(\theta(y))) \frac{\theta(x)-\theta(y)}{|x-y|^{1+\beta}} d x d y,
\end{aligned}
$$

which is clearly nonnegative since $F$ is a nondecreasing function.

Next we state and prove the local wellposedness and a continuation criterion for equation (1.1). The main issue is the nonlocal drift term $\Lambda^{-\alpha} u$ which induces some integrability constraints on $u$; see Remark 2.3 and Remark 2.4 below.

Theorem 2.2 (Local wellposedness and continuation criterion). Suppose $0<\alpha<$ $1, \nu \geq 0$ and $0 \leq \beta \leq 2$ in (1.1). Assume $1<p<\frac{1}{\alpha}$ and set $k_{0}=\max \left\{\frac{\frac{1}{p}-\frac{1}{2}}{\frac{1}{p}-\alpha}, 2\right\}$.

Suppose the initial data $u_{0} \in L^{p} \cap H^{k}$ for some integer $k>k_{0}$. Then there exists $T>0$ and a unique solution $u$ to (1.1) in the space $C\left([0, T), L^{p} \cap H^{k}\right)$ with $u(0)=$ $u_{0}$. The solution can be continued beyond any $T^{\prime} \geq T$ if

$$
\int_{0}^{T^{\prime}}\left\|\partial_{x} \Lambda^{-\alpha} u(t)\right\|_{\infty} d t<+\infty .
$$

Moreover if $\nu>0$ and $0<\beta \leq 2$, then the solution has additional regularity, i.e. $u \in C\left((0, T), L^{p} \cap H^{k^{\prime}}\right)$ for any $k^{\prime} \geq k$. 
Remark 2.3. In Theorem 2.2 to have local wellposedness, we require the initial data $u_{0}$ to lie in $L^{p}$ for some $1<p<\frac{1}{\alpha}$. For $0<\alpha \leq \frac{1}{2}$, one can just work with pure $H^{k}$ spaces. However, for $\frac{1}{2}<\alpha<1$, it is essential that $u_{0}$ belongs $L^{p}$ for some small $p$ in order to control the low frequency part. The regularity condition $k \geq k_{0}$ comes from bounding the quantity $\left\|\partial_{x} u\right\|_{\frac{1}{\alpha}}$ when we perform contraction estimates in $C_{t}^{0} L_{x}^{p}$ (see e.g. (2.8) ).

Remark 2.4. Instead of the space $L^{p} \cap H^{k}$, one can also choose the space $W^{k, p}$ for $1<p<\frac{1}{\alpha}$ when $\frac{1}{2}<\alpha<1$. Another possibility is the space $H^{-\delta} \cap H^{k}$ for some $\delta>0$. Of course, the choice of $k$ in Theorem 2.2 is not optimal. We shall not dwell on these issues here. In any case these spaces provide natural $L^{\infty}$ bounds on the drift term $\Lambda^{-\alpha} u$ and produce classical solutions.

Proof of Theorem 2.2. The proof is more or less a standard application of energy estimates, and hence we only sketch the details here. Define $u^{(0)}=u_{0}$, and for $n \geq 0$ inductively define the iterates $u^{(n+1)}$ such that

$$
\left\{\begin{array}{l}
\partial_{t} u^{(n+1)}=\left(\Lambda^{-\alpha} u^{(n)}\right) \partial_{x} u^{(n+1)}-\nu \Lambda^{\beta} u^{(n+1)}, \\
u^{(n+1)}(0)=u_{0} .
\end{array}\right.
$$

Note that the system (2.4) is linear in $u^{(n+1)}$. By a simple induction we have $u^{(n)} \in C\left([0, \infty), L^{p} \cap H^{k}\right)$ for all $n \geq 0$, and furthermore for some $T>0$ depending only on $u_{0}$,

$$
\sup _{n \geq 0}\left(\left\|u^{(n)}\right\|_{L_{t}^{\infty} H^{k}([0, T))}+\left\|u^{(n)}\right\|_{L_{t}^{\infty} L^{p}([0, T))}\right) \leq A_{1}<\infty,
$$

where $A_{1}>0$ is a constant. Note that in deriving (2.5), we have used (2.1).

The next step is to show contraction in $C\left(\left[0, T_{1}\right], L_{x}^{p}\right)$ for some $T_{1}$ possibly smaller than $T$. Define $w^{(n+1)}=u^{(n+1)}-u^{(n)}$. By (2.4), we have

$$
\partial_{t} w^{(n+2)}=\Lambda^{-\alpha} u^{(n+1)} \partial_{x} w^{(n+2)}+\Lambda^{-\alpha} w^{(n+1)} \partial_{x} u^{(n+1)}-\nu \Lambda^{\beta} w^{(n+2)} .
$$

Multiplying both sides by $\left|w^{(n+2)}\right|^{p-2} w^{(n+2)}$, integrating by parts and using (2.1) again, we have

$$
\begin{aligned}
\frac{1}{p} \frac{d}{d t} \int_{\mathbb{R}}\left|w^{(n+2)}\right|^{p} d x \leq & \frac{1}{p} \int_{\mathbb{R}}\left|\partial_{x} \Lambda^{-\alpha} u^{(n+1)}\right|\left|w^{(n+2)}\right|^{p} d x \\
& +\int_{\mathbb{R}}\left|\Lambda^{-\alpha} w^{(n+1)}\right|\left|w^{(n+2)}\right|^{p-1}\left|\partial_{x} u^{(n+1)}\right| d x .
\end{aligned}
$$

By Hölder and Sobolev embedding,

$$
\begin{aligned}
\frac{d}{d t}\left(\left\|w^{(n+2)}(t)\right\|_{p}^{p}\right) \lesssim & \left\|\partial_{x} \Lambda^{-\alpha} u^{(n+1)}(t)\right\|_{\infty}\left\|w^{(n+2)}(t)\right\|_{p}^{p} \\
& +\left\|\Lambda^{-\alpha} w^{(n+1)}(t)\right\|_{\left(\frac{1}{p}-\alpha\right)^{-1}}\left\|w^{(n+2)}(t)\right\|_{p}^{p-1}\left\|\partial_{x} u^{(n+1)}(t)\right\|_{\frac{1}{\alpha}} \\
& \lesssim\left\|u^{(n+1)}(t)\right\|_{H^{2}}\left\|w^{(n+2)}(t)\right\|_{p}^{p} \\
& +\left\|w^{(n+1)}(t)\right\|_{p} \cdot\left\|w^{(n+2)}(t)\right\|_{p}^{p-1} \cdot\left\|\partial_{x} u^{(n+1)}(t)\right\|_{\frac{1}{\alpha}} .
\end{aligned}
$$

To bound $\left\|\partial_{x} u^{(n+1)}(t)\right\|_{\frac{1}{\alpha}}$ we discuss two cases. If $0<\alpha \leq \frac{1}{2}$, then one can use the Sobolev embedding to get

$$
\left\|\partial_{x} u^{(n+1)}(t)\right\|_{\frac{1}{\alpha}} \lesssim\left\|u^{(n+1)}(t)\right\|_{H^{2}} .
$$


If $\frac{1}{2}<\alpha<1$, then we use the interpolation inequality

to get

$$
\|g\|_{\frac{1}{\alpha}} \lesssim\|g\|_{p}^{1-\theta}\|g\|_{2}^{\theta}, \quad \theta=\frac{\frac{1}{p}-\alpha}{\frac{1}{p}-\frac{1}{2}},
$$

$$
\begin{aligned}
\left\|\partial_{x} u^{(n+1)}\right\|_{\frac{1}{\alpha}} & \lesssim\left\|u^{(n+1)}\right\|_{p}+\sum_{N>1} N\left\|P_{N} u^{(n+1)}\right\|_{p}^{1-\theta}\left\|P_{N} u^{(n+1)}\right\|_{2}^{\theta} \\
& \lesssim\left\|u^{(n+1)}\right\|_{p}+\left\|u^{(n+1)}\right\|_{H^{k}},
\end{aligned}
$$

where we have used the fact that $k>k_{0} \geq \frac{1}{\theta}$. Plugging the bounds (2.7)-(2.8) into (2.6) and using (2.5), we obtain

$$
\frac{d}{d t}\left(\left\|w^{(n+2)}(t)\right\|_{p}^{p}\right) \lesssim A_{1} \cdot\left(\left\|w^{(n+2)}(t)\right\|_{p}^{p}+\left\|w^{(n+1)}(t)\right\|_{p} \cdot\left\|w^{(n+2)}(t)\right\|_{p}^{p-1}\right) .
$$

By using the fact $w^{(n+1)}(0)=0$ for all $n \geq 0$, and choosing $T_{1}$ smaller than $T$ if necessary, we get for some $0<\tilde{\theta}<1$ that

$$
\left\|w^{(n+2)}(t)\right\|_{L_{t}^{\infty} L_{x}^{p}\left(\left[0, T_{1}\right]\right)} \leq \tilde{\theta}\left\|w^{(n+1)}(t)\right\|_{L_{t}^{\infty} L_{x}^{p}\left(\left[0, T_{1}\right]\right)}, \quad \forall n \geq 0 .
$$

Hence the sequence $u^{(n)}$ has a strong limit in $C\left(\left[0, T_{1}\right], L_{x}^{p}\right)$.

By interpolation one also obtains strong convergence and the limit solution $u$ in $C\left(\left[0, T_{1}\right], H^{k-1}\right)$. By a standard argument (one has to discuss separately the case $\nu=0$ and the case $\nu>0$, and the fact that strong continuity in $H^{k}$ is equivalent to weak continuity together with norm continuity), one can show that $u \in C\left(\left[0, T_{1}\right], H^{k}\right)$. We omit the details.

Now we turn to the proof of the continuation criterion (2.3). By (2.1), it is easy to show that

$$
\frac{d}{d t}\left(\|u(t)\|_{p}^{p}\right) \lesssim\left\|\partial_{x} \Lambda^{-\alpha} u(t)\right\|_{\infty}\|u(t)\|_{p}^{p},
$$

and hence $\|u(t)\|_{p}$ is controlled by (2.3). It remains to control the $H^{k}$ norm. From (1.1), using integration by parts and Hölder, we have

$$
\begin{aligned}
& \frac{d}{d t}\left(\left\|\partial_{x}^{k} u(t)\right\|_{2}^{2}\right) \\
& \lesssim \int_{\mathbb{R}} \partial_{x}^{k}\left(\Lambda^{-\alpha} u \partial_{x} u\right) \partial_{x}^{k} u d x \\
& \lesssim\left\|\partial_{x} \Lambda^{-\alpha} u(t)\right\|_{\infty}\left\|\partial_{x}^{k} u(t)\right\|_{2}^{2} \\
& \quad+\left\|\partial_{x}^{k} u(t)\right\|_{2} \sum_{1 \leq l \leq k}\left\|\partial_{x}^{l-1} \partial_{x} \Lambda^{-\alpha} u(t)\right\|_{\frac{2(k-1+\alpha)}{l-1}} \cdot\left\|\partial_{x}^{k-l} \Lambda^{\alpha} \partial_{x} \Lambda^{-\alpha} u\right\|_{\frac{2(k-1+\alpha)}{k-l+\alpha}}
\end{aligned}
$$

By using the Gagliardo-Nirenberg inequalities, we have for any $1 \leq l \leq k$,

$$
\begin{aligned}
\left\|\partial_{x}^{l-1} \partial_{x} \Lambda^{-\alpha} u(t)\right\|_{\frac{2(k-1+\alpha)}{l-1}} \lesssim\left\|\partial_{x} \Lambda^{-\alpha} u(t)\right\|_{\infty}^{1-\frac{l-1}{k-1+\alpha}} \cdot\left\|\partial_{x}^{k} u(t)\right\|_{2}^{\frac{l-1}{k-1+\alpha}}, \\
\left\|\partial_{x}^{k-l} \Lambda^{\alpha} \partial_{x} \Lambda^{-\alpha} u\right\|_{\frac{2(k-1+\alpha)}{k-l+\alpha}} \lesssim\left\|\partial_{x} \Lambda^{-\alpha} u\right\|_{\infty}^{1-\frac{k-l+\alpha}{k-1+\alpha}} \cdot\left\|\partial_{x}^{k} u\right\|_{2}^{\frac{k-l+\alpha}{k-1+\alpha}} .
\end{aligned}
$$

Plugging the above estimates into (2.10), we obtain

$$
\frac{d}{d t}\left(\left\|\partial_{x}^{k} u(t)\right\|_{2}^{2}\right) \lesssim\left\|\partial_{x} \Lambda^{-\alpha} u(t)\right\|_{\infty} \cdot\left\|\partial_{x}^{k} u(t)\right\|_{2}^{2}
$$

Together with (2.9) this easily yields (2.3). 
Finally in the case $\nu>0$ and $0<\beta \leq 2$, one can use the theory of mild solutions or simple energy estimates to gain additional regularity. See, for instance, [10]. We omit the details.

Remark 2.5. From the logarithmic type bound

$$
\left\|\partial_{x} \Lambda^{-1} f\right\|_{L^{\infty}} \leq C\left[1+\|f\|_{L^{\infty}} \log \left(e+\|f\|_{H^{1}}\right)+\|f\|_{L^{2}}\right],
$$

it is easily seen that condition (2.3) can be replaced by

$$
\int_{0}^{T^{\prime}}\left\|\Lambda^{1-\alpha} u(t)\right\|_{\infty} d t<\infty .
$$

However, we shall not use this fact in the sequel.

For the proof of Theorem 1.2, when $\beta<2$ we shall use the idea of the nonlocal maximum principle for a suitably chosen modulus of continuity. This method was first used by Kiselev, Nazarov, and Volberg in [15, where they proved the global regularity for the $2 \mathrm{D}$ critical dissipative quasi-geostrophic equations with periodic $C^{\infty}$ data. In the borderline case when $\beta=2$, this argument does not seem to work since (2.14) below no longer holds. Therefore, we will use a different approach in this case.

We say a function $f$ has modulus of continuity $\omega$ if $|f(x)-f(y)| \leq \omega(|x-y|)$, where $\omega$ is an increasing continuous function $\omega:[0,+\infty) \rightarrow[0,+\infty)$ and $\omega(0)=0$. We say $f$ has strict modulus of continuity $\omega$ if the inequality is strict for $x \neq y$.

In what follows, we will choose a concave function $\omega$ satisfying

$$
\omega^{\prime}>0, \quad \omega^{\prime}(0)<+\infty, \quad \lim _{\xi \rightarrow 0^{+}} \omega^{\prime \prime}(\xi)=-\infty .
$$

Owing to Theorem 2.2 and the Sobolev embedding theorem, we may assume $\theta_{0} \in H^{20} \cap C^{\infty}$. Because of the scaling property of (1.1), for any $\lambda>0$,

$$
u_{\lambda}(t, x)=\lambda^{\alpha+\beta-1} u\left(\lambda^{\beta} t, \lambda x\right)
$$

is also a solution of (1.1) with initial data $\lambda^{\alpha+\beta-1} u_{0}\left(\lambda^{\beta} x\right)$. Thus if we can show that $u_{\lambda}$ is a global solution, the same remains true for $u$. Note that for any $\omega$ satisfying (2.11) we can always find a constant $\lambda>0$ such that $\omega(\xi)$ is a strict modulus of continuity of $\lambda^{\alpha+\beta-1} u_{0}\left(\lambda^{\beta} x\right)$ provided that $\alpha+\beta>1$. While in the critical case, i.e., $\alpha+\beta=1$, this still holds for any unbounded $\omega$ satisfying (2.11).

We shall show that for suitably chosen $\omega$, the modulus of continuity is preserved for all of the time. This gives $\left\|\partial_{x} u(t)\right\|_{\infty} \leq \omega^{\prime}(0)$, which together with the uniform boundedness of $\|u(t)\|_{\infty}$ and Theorem 2.2 implies Theorem 1.2. Following the argument in [15] and [11, the strict modulus of continuity is preserved, at least for a short time. Also it is clear that if $u(t, \cdot)$ has strict modulus of continuity $\omega$ for all $t \in[0, T)$, then $\theta$ is smooth up to $T$ and $u(T, \cdot)$ has modulus of continuity $\omega$ by continuity. Therefore, to show that the modulus of continuity is preserved for all of the time, it suffices to rule out the case that

$$
\sup _{x \neq y} \frac{u(T, x)-u(T, y)}{\omega(|x-y|)}=1
$$

which in turn implies that there exist two different points $x, y \in \mathbb{R}$ satisfying

$$
u(T, x)-u(T, y)=\omega(|x-y|)
$$


due to (2.11) and the decay of $u$ at the spatial infinity (see [11). This possibility can be ruled out eventually if we are able to choose suitable $\omega$ such that under the conditions above we have

$$
\frac{\partial}{\partial t}(u(T, x)-u(T, y))<0 .
$$

To this end, we need the following two lemmas.

Lemma 2.6. Assume that $u$ has modulus of continuity $\omega$, which is a concave function. Then for any $\alpha \in(0,1), \Lambda^{-\alpha} u$ has modulus of continuity

$$
\Omega(r):=C_{\alpha}\left(r^{\alpha} \omega(r)+r \int_{r}^{\infty} \frac{w(s)}{s^{2-\alpha}} d s\right) .
$$

Proof. For any $r>0$, take $x, y \in \mathbb{R}$ such that $|x-y|=r$. Without loss of generality, we may assume $x=-r / 2$ and $y=r / 2$. Then

$$
\begin{gathered}
\left|\Lambda^{-\alpha} u(x)-\Lambda^{-\alpha} u(y)\right|=C_{\alpha}\left|\int_{-\infty}^{\infty} \frac{u(s)-u(0)}{|r / 2+s|^{1-\alpha}} d s-\int_{-\infty}^{\infty} \frac{u(s)-u(0)}{|-r / 2+s|^{1-\alpha}} d s\right| \\
\leq I_{1}+I_{2} .
\end{gathered}
$$

Here

$$
I_{1}:=C_{\alpha}\left|\int_{-r}^{r} \frac{u(s)-u(0)}{|r / 2+s|^{1-\alpha}} d s\right|+C_{\alpha}\left|\int_{-r}^{r} \frac{u(s)-u(0)}{|-r / 2+s|^{1-\alpha}} d s\right| \leq C_{\alpha} r^{\alpha} \omega(r)
$$

and

$$
\begin{aligned}
I_{2} & :=C_{\alpha}\left(\int_{-\infty}^{-r}+\int_{r}^{\infty}\right)|u(s)-u(0)|\left|\frac{1}{|r / 2+s|^{1-\alpha}}-\frac{1}{|-r / 2+s|^{1-\alpha}}\right| \\
& \leq C_{\alpha} r \int_{r}^{\infty} \frac{w(s)}{s^{2-\alpha}} d s
\end{aligned}
$$

where in the last inequality we have used the mean value theorem. The lemma is proved.

Lemma 2.7. Assume $u$ and $\Lambda^{-\alpha} u$ have moduli of continuity $\omega$ and $\Omega$ respectively, and $u(x)-u(y)=\omega(|x-y|)$ for some $x \neq y$.

i) Then we have

$$
\left|\Lambda^{-\alpha} u(x) \partial_{x} u(x)-\Lambda^{-\alpha} u(y) \partial_{x} u(y)\right| \leq \Omega(|x-y|) \omega^{\prime}(|x-y|) .
$$

ii) For any $\beta \in(0,2)$, it holds that

$$
\begin{aligned}
-\Lambda^{\beta} u(x)+\Lambda^{\beta} u(y) \leq & C_{\beta} \int_{0}^{\frac{r}{2}} \frac{\omega(r+2 s)+\omega(r-2 s)-2 \omega(r)}{r^{1+\beta}} d s \\
& +C_{\beta} \int_{\frac{r}{2}}^{\infty} \frac{\omega(2 s+r)-\omega(2 s-r)-2 \omega(r)}{r^{1+\beta}} d s,
\end{aligned}
$$

where $r=|x-y|$.

Proof. See [15], or [10]. 
Due to Lemmas 2.6 and 2.7, the left-hand side of (2.12) is less than or equal to $I_{3}+I_{4}+I_{5}$, where

$$
\begin{gathered}
I_{3}=\Omega(r) \omega^{\prime}(r), \quad I_{4}=C_{\beta} \int_{0}^{\frac{r}{2}} \frac{\omega(r+2 s)+\omega(r-2 s)-2 \omega(r)}{r^{1+\beta}} d s, \\
I_{5}=C_{\beta} \int_{\frac{r}{2}}^{\infty} \frac{\omega(2 s+r)-\omega(2 s-r)-2 \omega(r)}{r^{1+\beta}} d s, \quad r=|x-y|,
\end{gathered}
$$

where $\Omega(r)$ is given in (2.13). With concave $\omega$, both $I_{4}$ and $I_{5}$ are strictly negative.

Now we are ready to prove Theorem 1.2 .

Proof of Theorem 1.2. Let $\delta>0$ be a small number to be specified later. We consider three cases separately.

Case 1: $\beta=1-\alpha$. Set for $r>0$,

$$
\omega^{\prime \prime}(r)=-\frac{\delta}{r^{\alpha}+r^{2}}, \quad \omega^{\prime}(r)=-\int_{r}^{\infty} \omega^{\prime \prime}(s) d s, \quad \omega(0)=0,
$$

which clearly satisfies (2.11). Since $\alpha \in(0,1)$, it is easily seen that, for $r \ll 1$,

$$
\begin{aligned}
\omega^{\prime}(r) & \sim \delta, \quad \omega(r) \sim \delta r, \quad \Omega(r) \sim \delta r, \\
I_{3}=\Omega \omega^{\prime} \sim \delta^{2} r, \quad I_{4} & \leq C \delta r^{2-\beta} \omega^{\prime \prime}(r) \sim-\delta r^{2-\beta-\alpha} .
\end{aligned}
$$

The last inequality is because of the mean value theorem and the monotonicity of $\omega^{\prime \prime}$. Similarly, for $r \gg 1$,

$$
\begin{aligned}
\omega^{\prime}(r) & \sim \delta r^{-1}, \quad \omega(r) \sim \delta \log r, \quad \Omega(r) \sim \delta r^{\alpha} \log r, \\
I_{3} & =\Omega \omega^{\prime} \sim \delta^{2} r^{\alpha-1} \log r, \quad I_{5} \leq-C \delta r^{-\beta} \log r .
\end{aligned}
$$

For the bound of $I_{5}$, we have used $\omega(2 s+r)-\omega(2 s-r) \leq \omega(2 r)$. Recall that $\alpha+\beta=$ 1. Here and in what follows, the constant $C$ is independent of $\delta$. From (2.15), (2.16) and the continuity, we can choose $\delta$ sufficiently small such that $I_{3}+I_{4}+I_{5}$ is strictly negative on $(0,+\infty)$. Thus, we obtain (2.12).

Case 2: $\beta \in(1-\alpha, 2)$. Set for $r>0$,

$$
\omega^{\prime \prime}(r)=-\frac{\delta}{r^{\alpha}+r^{5}}, \quad \omega^{\prime}(r)=-\int_{r}^{\infty} \omega^{\prime \prime}(s) d s, \quad \omega(0)=0,
$$

which clearly satisfies (2.11). We still have (2.15) for $r \ll 1$. For $r \gg 1$,

$$
\begin{aligned}
\omega^{\prime}(r) & \sim \delta r^{-4}, \quad \omega(r) \sim \delta, \quad \Omega(r) \sim \delta r^{\alpha}, \\
I_{3} & =\Omega \omega^{\prime} \sim \delta^{2} r^{\alpha-4}, \quad I_{5} \leq-C \delta r^{-\beta} .
\end{aligned}
$$

Recall that $\alpha+\beta \in(1,4)$. From (2.15), (2.17) and the continuity, we can choose $\delta$ sufficiently small such that $I_{3}+I_{4}+I_{5}$ is strictly negative on $(0,+\infty)$. Therefore, we still obtain (2.12) in this case.

Case 3: $\beta=2$. In this case, we shall use a different argument since the bound (2.14) does not hold any more. Due to the classical Sobolev theory for secondorder parabolic equations 1 it suffices for us to show that

$$
\left\|\Lambda^{-\alpha} u\right\|_{L_{t}^{\infty} L_{x}^{\infty}([0, T])}<\infty
$$

\footnotetext{
${ }^{1}$ See also Remark 2.8 for a different proof.
} 
for any finite $T>0$. Indeed, by the classical theory (2.18) guarantees that $u, \partial_{x} u, \partial_{x x} u, \partial_{t} u \in L^{2}([0, T] \times \mathbb{R})$, which together with the Sobolev imbedding theorem implies (2.3).

In order to prove (2.18), first we note that by the maximum principle, for any $t>0$

$$
\|u(t)\|_{\infty} \leq\|u(0)\|_{\infty} .
$$

Using the Littlewood-Paley projectors, the Bernstein inequality, and (2.19), we have

$$
\begin{aligned}
\left\|\Lambda^{-\alpha} u(t)\right\|_{\infty} & \lesssim\|u(t)\|_{\infty}+\sum_{\mathrm{N} \text { dyadic: } N \leq 1}\left\|\Lambda^{-\alpha} P_{N} u(t)\right\|_{\infty} \\
& \lesssim\|u(0)\|_{\infty}+\sum_{N \leq 1} N^{-\alpha} N^{\frac{1}{p}}\|u(t)\|_{p} \\
& \lesssim\|u(0)\|_{\infty}+\|u(t)\|_{p},
\end{aligned}
$$

where $p$ is from Theorem 2.2, and in the third inequality we used the fact that $\alpha<\frac{1}{p}$. Multiplying both sides of (1.1) by $|u|^{p-2} u$ and integrating by parts, we have

$$
\begin{aligned}
\frac{1}{p} \frac{d}{d t} \int_{\mathbb{R}}|u|^{p} d x & =\int_{\mathbb{R}} \Lambda^{-\alpha} u \partial_{x} u|u|^{p-2} u d x+\nu \int_{\mathbb{R}} \partial_{x x} u|u|^{p-2} u d x \\
& =I_{1}+I_{2}-\frac{4 \nu(p-1)}{p^{2}} \int_{\mathbb{R}}\left(\partial_{x}|u|^{\frac{p}{2}}\right)^{2} d x,
\end{aligned}
$$

where

$$
I_{1}:=\int_{\mathbb{R}}\left(P_{<1} \Lambda^{-\alpha} u\right) \partial_{x} u|u|^{p-2} u d x, \quad I_{2}:=\int_{\mathbb{R}}\left(P_{\geq 1} \Lambda^{-\alpha} u\right) \partial_{x} u|u|^{p-2} u d x .
$$

To estimate $I_{1}$, we integrate by parts and use Hölder's inequality, the Bernstein inequality, and (2.19) to get

$$
\begin{aligned}
\left|I_{1}\right| & \leq \int_{\mathbb{R}}\left|P_{<1} \partial_{x} \Lambda^{-\alpha} u \| u\right|^{p} d x \\
& \leq\left\|P_{<1} \partial_{x} \Lambda^{-\alpha} u(t)\right\|_{p}\|u(t)\|_{p}^{p-1}\|u(t)\|_{\infty} \leq C\|u(t)\|_{p}^{p} .
\end{aligned}
$$

To bound $I_{2}$, by Hölder's inequality, the Bernstein inequality, and (2.19) we have

$$
\begin{aligned}
\left|I_{2}\right| & \leq C\left\|P_{\geq 1} \Lambda^{-\alpha} u(t)\right\|_{\infty}\left\|\partial_{x}|u|^{\frac{p}{2}}\right\|_{2}\left\||u|^{\frac{p}{2}}\right\|_{2} \\
& \leq C\|u(t)\|_{\infty}\left\|\partial_{x}|u|^{\frac{p}{2}}\right\|_{2}\left\||u|^{\frac{p}{2}}\right\|_{2} \\
& \leq C\|u(0)\|_{\infty}\left\|\partial_{x}|u|^{\frac{p}{2}}\right\|_{2}\left\||u|^{\frac{p}{2}}\right\|_{2} .
\end{aligned}
$$

Combining (2.21), (2.22), (2.23), and using Young's inequality and Gronwall's inequality, we easily get $\|u(t)\|_{p} \leq C e^{C t}$, which together with (2.20) yields (2.18).

The theorem is proved.

Remark 2.8. A slightly different proof for $\beta=2$ is possible, and we sketch it below for the sake of completeness. Multiplying both sides of (1.1) by $-\partial_{x x} u$ and 
integrating by parts, we have

$$
\begin{aligned}
& \frac{1}{2} \frac{d}{d t} \int\left(\partial_{x} u\right)^{2} d x \\
& =-\int\left(P_{<1} \Lambda^{-\alpha} u\right) \frac{1}{2} \partial_{x}\left(\left(\partial_{x} u\right)^{2}\right) d x-\int P_{\geq 1} \Lambda^{-\alpha} u \cdot \partial_{x} u \partial_{x x} u d x-\nu \int\left(\partial_{x x} u\right)^{2} d x \\
& =\frac{1}{2} \int \partial_{x} P_{<1} \Lambda^{-\alpha} u \cdot\left(\partial_{x} u\right)^{2} d x-\int P_{\geq 1} \Lambda^{-\alpha} u \cdot \partial_{x} u \partial_{x x} u d x-\nu \int\left(\partial_{x x} u\right)^{2} d x \\
& \lesssim\|u(t)\|_{\infty}\left\|\partial_{x} u(t)\right\|_{2}^{2}+\frac{2}{\nu}\left\|P_{\geq 1} \Lambda^{-\alpha} u(t)\right\|_{\infty}^{2}\left\|\partial_{x} u(t)\right\|_{2}^{2}-\frac{\nu}{2}\left\|\partial_{x x} u(t)\right\|_{2}^{2} \\
& \lesssim\left(\frac{2}{\nu}+1\right)\left(\|u(t)\|_{\infty}^{2}+1\right)\left\|\partial_{x} u(t)\right\|_{2}^{2}-\frac{\nu}{2}\left\|\partial_{x x} u(t)\right\|_{2}^{2} .
\end{aligned}
$$

Since $\|u(t)\|_{\infty} \leq\left\|u_{0}\right\|_{\infty}$, a Gronwall in time argument then yields

$$
\left\|\partial_{x} u(t)\right\|_{2}^{2}+\nu \int_{0}^{T}\left\|\partial_{x x} u\right\|_{2}^{2} d t \lesssim e^{C t}, \quad \forall t \geq 0 .
$$

Now global wellposedness quickly follows from the continuation criterion (2.3) and (2.24), since

$$
\begin{aligned}
\left\|\partial_{x} \Lambda^{-\alpha} u(t)\right\|_{\infty} & \lesssim\left\|P_{<1} \partial_{x} \Lambda^{-\alpha} u(t)\right\|_{\infty}+\left\|P_{\geq 1} \partial_{x} \Lambda^{-\alpha} u(t)\right\|_{\infty} \\
& \lesssim\|u(t)\|_{\infty}+\sum_{N \geq 1} N^{1-\alpha+\frac{1}{2}-2}\left\|\partial_{x x} u(t)\right\|_{2} \\
& \lesssim\left\|u_{0}\right\|_{\infty}+\left\|\partial_{x x} u(t)\right\|_{2} .
\end{aligned}
$$

Remark 2.9. The above arguments can be modified to prove to the global wellposedness of the following $1 \mathrm{D}$ model when $\beta=2$ :

$$
\partial_{t} u=H u \partial_{x} u-\nu \Lambda^{\beta} u,
$$

where $\nu>0$ is a constant. This equation has been studied recently in [7, and later in 10,18 . It is now known that when $\beta \in[1,2)$ the equation is globally well-posed. While in the range $\beta \in[0,1 / 4)$, evolving from a family of initial data solutions blow up in finite time (see [18). In [7, an additional positivity assumption is imposed on $u_{0}$. On the other hand, the proof of the global wellposedness in [10] relies on the nonlocal maximum principle, which does not work when $\beta=2$ by the same reasoning as above.

To deal with this borderline case, we multiply both sides of (2.26) by $u$ and integrate by parts to get

$$
\begin{aligned}
\frac{1}{2} \frac{d}{d t} \int_{\mathbb{R}} u^{2} d x & =\int_{\mathbb{R}} H u\left(\partial_{x} u\right) u d x+\nu \int_{\mathbb{R}}\left(\partial_{x x} u\right) u d x \\
& =-\frac{1}{2} \int_{\mathbb{R}}\left(\partial_{x} H u\right) u^{2} d x-\nu \int_{\mathbb{R}}\left(\partial_{x} u\right)^{2} d x \\
& \leq\left\|\partial_{x} H u(t)\right\|_{2}\|u(t)\|_{2}\|u(t)\|_{\infty}-\nu \|\left(\partial_{x} u(t) \|_{2}^{2}\right. \\
& \leq\left\|\partial_{x} u(t)\right\|_{2}\|u(t)\|_{2}\|u(0)\|_{\infty}-\nu \|\left(\partial_{x} u(t) \|_{2}^{2},\right.
\end{aligned}
$$

where in the last inequality we use the boundedness of the Hilbert transform in $L^{2}$ and the maximum principle. As before, by Young's inequality and Gronwall's inequality, we get $\|u(t)\|_{2} \leq C e^{C t}$, which further implies that $\|H u(t)\|_{2} \leq C e^{C t}$. Therefore, by the classical Sobolev theory, $u$ is globally regular. 
Alternatively, we multiply both sides of $(2.26)$ by $-\partial_{x x} u$ and integrate by parts to get

$$
\begin{aligned}
& \frac{1}{2} \frac{d}{d t} \int\left(\partial_{x} u\right)^{2} d x \\
& =-\int(H u) \frac{1}{2} \partial_{x}\left(\left(\partial_{x} u\right)^{2}\right) d x-\nu \int\left(\partial_{x x} u\right)^{2} d x \\
& =\frac{1}{2} \int\left(H \partial_{x} u\right)\left(\partial_{x} u\right)^{2} d x-\nu \int\left(\partial_{x x} u\right)^{2} d x \\
& =-\frac{1}{2} \int\left(H \partial_{x x} u \partial_{x} u+H \partial_{x} u \partial_{x x} u\right) u d x-\nu \int\left(\partial_{x x} u\right)^{2} d x \\
& \leq\left\|\partial_{x x} u(t)\right\|_{2}\left\|\partial_{x} u(t)\right\|_{2}\|u(t)\|_{\infty}-\nu\left\|\partial_{x x} u(t)\right\|_{2}^{2} .
\end{aligned}
$$

Since $\|u(t)\|_{\infty} \leq\left\|u_{0}\right\|_{\infty}$, by Young's inequality and Gronwall's inequality, we get

$$
\left\|\partial_{x} u(t)\right\|_{2}^{2}+\nu \int_{0}^{T}\left\|\partial_{x x} u\right\|_{2}^{2} d t \leq C e^{C t}, \quad \forall t \geq 0 .
$$

Note that (2.25) still holds when $\alpha=0$, which implies the global regularity of $u$ by the Beale-Kato-Majda criterion.

\section{Auxiliary lemmas}

This section is devoted to several auxiliary lemmas, which will be used in the proofs of Proposition 1.3 and Theorem 1.1 in the following section.

Lemma 3.1. Let $0<\alpha<1$. Assume $0<\delta<2$ and

$$
g(x)=|x|^{-\delta} \operatorname{sgn}(x)=\left\{\begin{array}{l}
x^{-\delta}, \quad \text { if } x>0, \\
-|x|^{-\delta}, \quad \text { if } x<0 .
\end{array}\right.
$$

Then

$$
\left(\Lambda^{-\alpha} g\right)(x)=C_{\alpha, \delta}|x|^{\alpha-\delta} \operatorname{sgn}(x),
$$

where $C_{\alpha, \delta}>0$ is a constant depending only on $(\alpha, \delta)$. Similarly for $0 \leq \beta<1$, we have

$$
\left(\Lambda^{\beta} g\right)(x)=C_{\beta, \delta}|x|^{-\delta-\beta} \operatorname{sgn}(x),
$$

where $C_{\beta, \delta}>0$ is another constant depending only on $(\beta, \delta)$.

Proof of Lemma 3.1. We first note that for any odd function $f=f(y)$,

$$
\left(\Lambda^{-\alpha} f\right)(x)=C_{\alpha} \int_{0}^{\infty} f(y)\left(\frac{1}{|x-y|^{1-\alpha}}-\frac{1}{|x+y|^{1-\alpha}}\right) d y
$$

where $C_{\alpha}>0$ is a constant depending only on $\alpha$.

By a simple scaling argument, we then have for $x>0$,

$$
\left(\Lambda^{-\alpha} g\right)(x)=C_{\alpha, \delta}^{\prime} x^{\alpha-\delta} \int_{0}^{\infty} y^{-\delta}\left(\frac{1}{|1-y|^{1-\alpha}}-\frac{1}{(1+y)^{1-\alpha}}\right) d y
$$

where $C_{\alpha, \delta}^{\prime}>0$ is a constant. It is easy to check that the integral in the last equality converges for $0<\alpha<1,0<\delta<2$. The identity (3.1) follows easily. 
Next we prove (3.2). Without loss of generality, we can assume $0<\beta<1$. Note that for any odd function $f$, we have

$$
\left(\Lambda^{\beta} f\right)(x)=C_{\beta} \int_{0}^{\infty}\left(\frac{f(x)-f(y)}{|x-y|^{1+\beta}}+\frac{f(x)+f(y)}{|x+y|^{1+\beta}}\right) d y .
$$

Again by a scaling argument, we have for $x>0$,

$$
\left(\Lambda^{\beta} g\right)(x)=C_{\beta, \delta} x^{-\delta-\beta} \int_{0}^{\infty}\left(\frac{1-y^{-\delta}}{|1-y|^{1+\beta}}+\frac{1+y^{-\delta}}{|1+y|^{1+\beta}}\right) d y .
$$

Since $0<\delta<2$ and $0<\beta<1$, it is not difficult to check that the last integral converges. The lemma is proved.

Lemma 3.2. Let $0<\alpha<\alpha_{1}<1$. Define $g$ by

$$
g(x)=|x|^{-\alpha_{1}} \operatorname{sgn}(x) \chi_{|x| \geq 1}=\left\{\begin{array}{l}
|x|^{-\alpha_{1}}, \quad x \geq 1, \\
-|x|^{-\alpha_{1}}, \quad x<-1, \\
0, \quad \text { otherwise. }
\end{array}\right.
$$

Then

$$
\left\|\Lambda^{-\alpha} g\right\|_{L_{x}^{\infty}(\mathbb{R})} \leq C_{\alpha, \alpha_{1}}
$$

where $C_{\alpha, \alpha_{1}}$ is a constant depending only on $\left(\alpha, \alpha_{1}\right)$.

Proof of Lemma 3.2. By using the Littlewood-Paley projectors and noting that $g \in L_{x}^{\infty} \cap L_{x}^{\frac{1}{\alpha_{1}}+}$, we compute

$$
\begin{aligned}
\left\|\Lambda^{-\alpha} g\right\|_{\infty} & \lesssim\|g\|_{\infty}+\sum_{\mathrm{N} \text { dyadic: } N \leq 1}\left\|\Lambda^{-\alpha} P_{N} g\right\|_{\infty} \\
& \lesssim\|g\|_{\infty}+\sum_{N \leq 1} N^{-\alpha} N^{\alpha_{1}-}\|g\|_{\frac{1}{\alpha_{1}}+} \\
& \lesssim\|g\|_{\infty}+\|g\|_{\frac{1}{\alpha_{1}}+} \\
& \leq C_{\alpha, \alpha_{1}},
\end{aligned}
$$

where in the second inequality we have used the Bernstein inequality. In the third inequality we used the fact that $\alpha<\alpha_{1}$ to make the summation over dyadic $N<1$ converge.

Lemma 3.3. Suppose $0<\alpha<1$. Assume $0<\theta<1-\alpha$. Then for $\lambda \in \mathbb{R}$, $g=g(x)=|x|^{i \lambda-\theta}=e^{(i \lambda-\theta) \log |x|}$, we have

$$
\left(\Lambda^{\alpha} g\right)(x)=2^{\alpha} \frac{\Gamma\left(\frac{1-\theta+i \lambda}{2}\right)}{\Gamma\left(\frac{\theta-i \lambda}{2}\right)} \cdot \frac{\Gamma\left(\frac{\theta+\alpha-i \lambda}{2}\right)}{\Gamma\left(\frac{1-\theta-\alpha+i \lambda}{2}\right)} \cdot|x|^{-(\theta+\alpha-i \lambda)}, \quad 0 \neq x \in \mathbb{R} .
$$

Here $\Gamma=\Gamma(z)$ is the usual Gamma function defined by

$$
\Gamma(z)=\int_{0}^{\infty} e^{-s} s^{z-1} d s, \quad \operatorname{Re}(z)>0 .
$$

Proof of Lemma 3.3. By using (3.5) and a simple change of variable argument, we have for $0 \neq x \in \mathbb{R}, z \in \mathbb{C}$ with $\operatorname{Re}(z)>0$,

$$
|x|^{-z}=\frac{1}{\Gamma\left(\frac{z}{2}\right)} \int_{0}^{\infty} e^{-s|x|^{2}} s^{\frac{1}{2} z-1} d s .
$$


By using (3.6) and the explicit form of the Fourier transform of Gaussian functions, it is not difficult to check that

$$
\int_{-\infty}^{\infty}|x|^{-z} e^{-i x \cdot \xi} d x=\sqrt{\pi} 2^{1-z} \cdot \frac{\Gamma\left(\frac{1-z}{2}\right)}{\Gamma\left(\frac{z}{2}\right)}|\xi|^{z-1}, \quad \forall 0 \neq \xi \in \mathbb{R}
$$

provided that $\operatorname{Re}(z) \in(0,1)$.

Take $z=\theta-i \lambda$; then clearly

$$
|x|^{-(\theta-i \lambda)}(\xi)=\sqrt{\pi} 2^{1-\theta+i \lambda} \frac{\Gamma\left(\frac{1-\theta+i \lambda}{2}\right)}{\Gamma\left(\frac{\theta-i \lambda}{2}\right)} \cdot|\xi|^{-(1-\theta+i \lambda)} .
$$

Consequently

$$
\left.|\xi|^{\alpha}\left|x \widehat{\mid-(\theta-i \lambda)}(\xi)=\sqrt{\pi} 2^{1-\theta+i \lambda} \frac{\Gamma\left(\frac{1-\theta+i \lambda}{2}\right)}{\Gamma\left(\frac{\theta-i \lambda}{2}\right)} \cdot\right| \xi\right|^{-(1-\theta-\alpha+i \lambda)} .
$$

Now note that $0<\theta+\alpha<1$. Using (3.7) again with $z=1-\theta-\alpha+i \lambda$, we get

$$
\mathcal{F}^{-1}\left(|\xi|^{\alpha} \widehat{|x|^{i \lambda-\theta}}(\xi)\right)(x)=2^{\alpha} \frac{\Gamma\left(\frac{1-\theta+i \lambda}{2}\right)}{\Gamma\left(\frac{\theta-i \lambda}{2}\right)} \cdot \frac{\Gamma\left(\frac{\theta+\alpha-i \lambda}{2}\right)}{\Gamma\left(\frac{1-\theta-\alpha+i \lambda}{2}\right)} \cdot|x|^{-(\theta+\alpha-i \lambda)} .
$$

This establishes (3.4).

The following lemma is crucial for the proof of Proposition 1.3.

Lemma 3.4. Suppose $0<\alpha<1$ and $0<\theta<1-\alpha$. Then for $\lambda \in \mathbb{R}, g=g(x)=$ $|x|^{i \lambda-\theta}$, we have

$$
-\left(\partial_{x} \Lambda^{\alpha} g\right)(x)=2^{\alpha+1} F_{\alpha, \theta}(\lambda) \cdot x^{-(\theta+\alpha+1-i \lambda)}, \quad x>0
$$

where

$$
F_{\alpha, \theta}(\lambda)=\frac{\Gamma\left(\frac{1-\theta+i \lambda}{2}\right)}{\Gamma\left(\frac{\theta-i \lambda}{2}\right)} \cdot \frac{\Gamma\left(1+\frac{\theta+\alpha-i \lambda}{2}\right)}{\Gamma\left(\frac{1-\theta-\alpha+i \lambda}{2}\right)},
$$

and also have the sharp bound

$$
\frac{1}{C_{\alpha, \theta}}\left(1+|\lambda|^{\alpha}\right) \leq \operatorname{Re}\left(F_{\alpha, \theta}(\lambda)\right) \leq C_{\alpha, \theta}\left(1+|\lambda|^{\alpha}\right), \quad \forall \lambda \in \mathbb{R} .
$$

Here $C_{\alpha, \theta}>0$ is a constant depending only on $(\alpha, \theta)$.

Proof of Lemma 3.4. Throughout this proof we will denote by the letter $C$ any constant which depends only on $(\alpha, \theta)$ but may vary from line to line. Note that (3.8) is a simple consequence of (3.4) and the fact that $z \Gamma(z)=\Gamma(z+1)$. We first establish the weaker bound

$$
\operatorname{Re}\left(F_{\alpha, \theta}(\lambda)\right) \geq \operatorname{Re}\left(F_{\alpha, \theta}(0)\right), \quad \forall \lambda \in \mathbb{R} .
$$

To show this we first differentiate $g$ and write

$$
-\partial_{x} g=(\theta-i \lambda)|x|^{i \lambda-\theta-1} \operatorname{sgn}(x) .
$$

Then using the fractional representation of $\Lambda^{\alpha}$ and a simple scaling argument, we have for any $x>0$,

$$
-\left(\Lambda^{\alpha} \partial_{x} g\right)(x)=C \cdot(\theta-i \lambda) x^{i \lambda-\theta-\alpha-1} \int_{0}^{\infty}\left(\frac{1-y^{i \lambda-\theta-1}}{|1-y|^{1+\alpha}}+\frac{1+y^{i \lambda-\theta-1}}{|1+y|^{1+\alpha}}\right) d y .
$$


Therefore

$$
\begin{aligned}
F_{\alpha, \theta}(\lambda)=C & \cdot(\theta-i \lambda) \cdot \int_{0}^{1}\left((1-y)^{-1-\alpha}\left(1+y^{\alpha-1}-y^{i \lambda-\theta-1}-y^{-i \lambda+\theta+\alpha}\right)\right. \\
& \left.\quad+(1+y)^{-1-\alpha}\left(1+y^{\alpha-1}+y^{i \lambda-\theta-1}+y^{-i \lambda+\theta+\alpha}\right)\right) d y \\
=: & C \cdot(\theta-i \lambda) G(\lambda) .
\end{aligned}
$$

By using the binomial expansion and a simple computation, we have

$$
\begin{array}{r}
G(\lambda) \\
=\sum_{\substack{k \geq 0, k \text { is even }}} C_{k, \alpha} \cdot\left(\frac{1}{k+1}+\frac{1}{k+\alpha}-\frac{k+\alpha+1}{k+1}\left(\frac{k+1-\theta}{(k+1-\theta)^{2}+\lambda^{2}}\right.\right. \\
\left.\left.+\frac{k+\theta+\alpha+2}{(k+\theta+\alpha+2)^{2}+\lambda^{2}}\right)\right) \\
\quad+i \lambda \sum_{\substack{k \geq 0, k \text { is even }}} C_{k+1, \alpha} \cdot\left(\frac{1}{(k+1-\theta)^{2}+\lambda^{2}}-\frac{1}{(k+\theta+\alpha+2)^{2}+\lambda^{2}}\right),
\end{array}
$$

where

$$
C_{k, \alpha}=(-1)^{k}\left(\begin{array}{c}
-\alpha-1 \\
k
\end{array}\right)=\frac{\Gamma(k+\alpha+1)}{\Gamma(k+1) \Gamma(\alpha+1)}=\frac{(\alpha+1) \cdots(\alpha+k)}{k !} .
$$

Using the asymptotics

$$
\left|\left(\begin{array}{c}
-\alpha-1 \\
k
\end{array}\right)\right| \sim \text { const } \cdot k^{\alpha}, \quad k \gtrsim 1,
$$

it is not difficult to check that the series representation of $G(\lambda)$ converges.

Clearly then

$$
\begin{aligned}
& \operatorname{Re}\left(F_{\alpha, \theta}(\lambda)\right) \\
& =\theta \sum_{\substack{k \geq 0, k \text { is even }}} C_{k, \alpha}\left(\frac{1}{k+1}+\frac{1}{k+\alpha}-\frac{k+\alpha+1}{k+1}\left(\frac{k+1-\theta}{(k+1-\theta)^{2}+\lambda^{2}}\right.\right. \\
& \left.+\lambda^{2} \sum_{\substack{k \geq 0, k \text { is even }}} C_{k+1, \alpha} \cdot\left(\frac{k+\theta+\alpha+2}{(k+\theta+\alpha+2)^{2}+\lambda^{2}}\right)\right)
\end{aligned}
$$

Owing to our assumption $0<\theta<1-\alpha, 0<\alpha<1$, it is easy to directly check from the above expression that (3.11) holds. Now by (3.9) and (3.11), we obtain

$$
\operatorname{Re}(F(\lambda)) \geq C>0, \quad \forall \lambda \in \mathbb{R} .
$$

It remains to prove the bound (3.10) for $\lambda$ sufficiently large. For this we need to use Stirling's formula for the Gamma function. It states that for any complex $z$ with $|\arg (z)|<\pi-\epsilon$ (here $\arg (z)$ takes values in $[-\pi, \pi))$ and $|z| \gg 1$, we have

$$
\ln \Gamma(z)=\left(z-\frac{1}{2}\right) \ln z-z+\frac{1}{2} \ln (2 \pi)+\frac{1}{12 z}+O\left(|z|^{-2}\right) .
$$


Using (3.9) and a tedious computation, we arrive at

$$
\ln F(\lambda)=O\left(\lambda^{-2}\right)+(1+\alpha) \ln \left(\frac{1}{2} \lambda\right)-i \frac{\pi}{2}+\frac{i}{4 \lambda}\left(\theta^{2}+4 \theta(1+\alpha)+2 \alpha(1+\alpha)\right),
$$

where $O\left(\lambda^{-2}\right)$ denotes the remainder term (complex-valued) whose absolute value is less than $\lambda^{-2}$. It follows easily that

$$
F(\lambda)=\left(\frac{1}{2} \lambda\right)^{1+\alpha}\left(-i \cos \left(\frac{C_{1}}{\lambda}\right)+\sin \left(\frac{C_{1}}{\lambda}\right)\right)+O\left(\lambda^{-1+\alpha}\right),
$$

where $C_{1}>0$ is a constant depending only on $(\alpha, \theta)$. Clearly

$$
\operatorname{Re}(F(\lambda))=C_{2} \lambda^{\alpha}+O\left(\lambda^{-1+\alpha}\right), \quad \lambda \gg 1,
$$

where $C_{2}>0$ is another constant. The sharp bound (3.10) follows.

\section{Finite-time Singularities}

In this section, we complete the proofs of Proposition 1.3 and Theorem 1.1. The proof of Proposition 1.3 is inspired by an argument in [7 by using Mellin transforms and the corresponding Parseval identity. See also [8, 12, 18, 19].

Proof of Proposition 1.3. Since by assumption $u$ is odd, using Lemma 3.1, we get

$$
\begin{aligned}
\int_{0}^{\infty} \frac{\Lambda^{-\alpha}\left(\Lambda^{-\alpha} u \partial_{x} u\right)}{x^{\delta}} d x & =\frac{1}{2} \int_{\infty}^{\infty} \Lambda^{-\alpha}\left(\Lambda^{-\alpha} u \partial_{x} u\right)|x|^{-\delta} \operatorname{sgn}(x) d x \\
& =\frac{1}{2} \int_{-\infty}^{\infty} \Lambda^{-\alpha} u \partial_{x} u \Lambda^{-\alpha}\left(|x|^{-\delta} \operatorname{sgn}(x)\right) d x \\
& =C_{\alpha, \delta} \int_{0}^{\infty} \Lambda^{-\alpha} u \cdot \partial_{x} u \cdot x^{\alpha-\delta} d x .
\end{aligned}
$$

By using the Parseval identity for Mellin transforms, we have

$$
\begin{aligned}
\int_{0}^{\infty} \Lambda^{-\alpha} u \partial_{x} u x^{\alpha-\delta} d x & =\int_{0}^{\infty} \frac{\Lambda^{-\alpha} u}{x^{\frac{\delta}{2}}} \cdot \frac{\partial_{x} u}{x^{\frac{\delta}{2}-\alpha-1}} \frac{d x}{x} \\
& =\frac{1}{2 \pi} \int_{-\infty}^{\infty} \overline{A(\lambda)} B(\lambda) d \lambda
\end{aligned}
$$

where

$$
\begin{aligned}
A(\lambda) & =\int_{0}^{\infty} \Lambda^{-\alpha} u \cdot x^{i \lambda-\frac{\delta}{2}-1} d x, \\
B(\lambda) & =\int_{0}^{\infty} \partial_{x} u \cdot x^{i \lambda-\frac{\delta}{2}+\alpha} d x \\
& =\int_{0}^{\infty} \partial_{x} \Lambda^{\alpha}\left(\Lambda^{-\alpha} u\right) \cdot x^{i \lambda-\frac{\delta}{2}+\alpha} d x .
\end{aligned}
$$

By Lemma 3.4 and observing that $\partial_{x} u$ is an even function on $\mathbb{R}$, we obtain (note here $\theta=\frac{\delta}{2}-\alpha$ and $\left.0<\theta<1-\alpha\right)$

$$
\begin{aligned}
B(\lambda) & =2^{\alpha+1} F_{\alpha, \theta}(\lambda) \int_{0}^{\infty}\left(\Lambda^{-\alpha} u\right) \cdot x^{-\left(\frac{\delta}{2}+1-i \lambda\right)} d x \\
& =2^{\alpha+1} F_{\alpha, \theta}(\lambda) A(\lambda)
\end{aligned}
$$


where $F_{\alpha, \theta}(\lambda)$ was defined in (3.9). Substituting (4.3) into (4.1) and using (3.10), we have

$$
\begin{aligned}
\int_{0}^{\infty} \Lambda^{-\alpha} u \partial_{x} u x^{\alpha-\delta} d x & =C_{\alpha, \theta} \int_{-\infty}^{\infty} \operatorname{Re}\left(F_{\alpha, \theta}(\lambda)\right)|A(\lambda)|^{2} d \lambda \\
& \geq C_{\alpha, \theta} \int_{-\infty}^{\infty}|A(\lambda)|^{2} d \lambda \\
& \geq C_{\alpha, \theta} \int_{0}^{\infty} \frac{\left(\Lambda^{-\alpha} u\right)^{2}}{x^{1+\delta}} d x
\end{aligned}
$$

where the last step follows from (4.2) and the Parseval identity for Mellin transforms. This completes the proof of (1.3).

Lemma 4.1 ( $L_{x}^{1}$ norm is nonincreasing). Assume $0<\alpha<1$ and $0 \leq \beta \leq 2$ in (1.1). Let the initial data $u_{0} \in L_{x}^{1}(\mathbb{R}) \cap H_{x}^{1}(\mathbb{R})$ be such that $u_{0}(x) \geq 0$ for any $x \geq 0$ and $u_{0}$ is odd in $x$. Let $u=u(t, x)$ be the corresponding solution to (1.1) with lifespan $\left[0, T_{0}\right)$ where $0<T_{0} \leq+\infty$. Then for any $t \in\left[0, T_{0}\right)$, we have $u(t, x) \geq 0$ for any $x \geq 0$, and

$$
\|u(t, \cdot)\|_{L_{x}^{1}(\mathbb{R})} \leq\left\|u_{0}\right\|_{L_{x}^{1}(\mathbb{R})} .
$$

Proof of Lemma 4.1. We shall use the idea of time-splitting approximation. Namely the solution $u=u(t, x)$ on any $\left[0, T^{\prime}\right]$ with $T^{\prime}<T_{0}$ can be approximated by interlacing the nonlinear evolution $\partial_{t} f=\Lambda^{-\alpha} f \partial_{x} f$ with the linear evolution $\partial_{t} g=-\nu \Lambda^{\beta} g$ with small time step $h=T^{\prime} / N$ where $N \rightarrow \infty$. First consider the linear evolution $\partial_{t} g=-\nu \Lambda^{\beta} g$ on a time interval $[0, h]$ with $g_{0}$ odd in $x$ and $g_{0}(x) \geq 0$ for any $x \geq 0$. Then clearly $g=g(t, x)$ is also odd in $x$ and has the representation

$$
g(t, x)=\int_{0}^{\infty}(k(t, x-y)-k(t, x+y)) g_{0}(y) d y,
$$

where $k(t, z)$ is the fundamental solution corresponding to the propagator $\partial_{t}+\nu \Lambda^{\beta}$, which is nonnegative and radially decreasing. It is easy to check that $g(t, x) \geq 0$ for any $x \geq 0$. Furthermore

$$
\begin{aligned}
\int_{0}^{\infty} g(t, x) d x & =\int_{0}^{\infty}\left(\int_{-y}^{y} k(t, z) d z\right) g_{0}(y) d y \\
& \leq \int_{0}^{\infty} g_{0}(y) d y
\end{aligned}
$$

where we used the fact that $k(t, z)$ is nonnegative and $\int_{-\infty}^{\infty} k(t, z) d z=1$. We now check the nonlinear evolution $\partial_{t} f=\Lambda^{-\alpha} f \partial_{x} f$ on the time interval $[0, h]$. Assume $f_{0}$ is odd in $x$ and $f_{0}(x) \geq 0$ for any $x \geq 0$. It is not difficult to check that $f(t, x)$ is odd in $x$ and $f(t, x) \geq 0$ for any $x \geq 0$. Then by using (3.3), integrating by parts, we compute (here for simplicity of presentation we omit any $\epsilon$-regularization 
argument needed for the convergence of integrals):

$$
\begin{aligned}
& \frac{d}{d t} \int_{0}^{\infty} f(t, x) d x \\
= & \int_{0}^{\infty} \Lambda^{-\alpha} f \partial_{x} f d x \\
= & \int_{0}^{\infty} \int_{0}^{\infty}\left(\partial_{x} f\right)(t, x) f(t, y) \cdot\left(|x-y|^{-(1-\alpha)}-|x+y|^{-(1+\alpha)}\right) d x d y \\
= & -\int_{0}^{\infty} \int_{0}^{\infty} f(t, x) f(t, y)\left(-(1-\alpha)|x-y|^{-(2-\alpha)} \operatorname{sgn}(x-y)\right. \\
& \left.\quad+(1+\alpha) \cdot|x+y|^{-(2+\alpha)}\right) d x d y \\
= & -\int_{0}^{\infty} \int_{0}^{\infty} f(t, x) f(t, y) \cdot(1+\alpha) \cdot|x+y|^{-(2+\alpha)} d x d y,
\end{aligned}
$$

where in the last equality we have used a symmetrization in $x$ and $y$ to make the first integral corresponding to the kernel $|x-y|^{-(2-\alpha)} \operatorname{sgn}(x-y)$ vanish. Hence we obtain $\|f(t, \cdot)\|_{L_{x}^{1}\left(\mathbb{R}^{+}\right)} \leq\left\|f_{0}\right\|_{L_{x}^{1}\left(\mathbb{R}^{+}\right)}$. This completes the proof for the nonlinear evolution part.

We are now ready to complete the

Proof of Theorem 1.1. We will argue by contradiction. Assume the solution corresponding to $u_{0}$ exists for all time. Then by Lemma 3.1, we have

$$
\begin{aligned}
\int_{0}^{\infty} \frac{\left(\Lambda^{-\alpha} u\right)(t, x)}{x^{\delta}} d x & =C_{\alpha, \delta} \int_{0}^{\infty} \frac{u(t, x)}{x^{\delta-\alpha}} d x \\
& \lesssim\|u(t, \cdot)\|_{L_{x}^{1}}+\int_{0}^{1} \frac{\left\|\partial_{x} u\right\|_{\infty}}{x^{\delta-\alpha-1}} d x \\
& \lesssim\|u(t, \cdot)\|_{L_{x}^{1}}+\left\|\partial_{x} u\right\|_{L_{x}^{\infty}}<+\infty .
\end{aligned}
$$

This shows that the integral $\int_{0}^{\infty}\left(\Lambda^{-\alpha} u\right)(t, x) x^{-\delta} d x$ is finite for any $t \geq 0$. Next we show that the integral blows up at some finite $T>0$ and we obtain a contradiction.

By using (1.1), Proposition 1.3 and Lemma 3.1, we have

$$
\begin{aligned}
\frac{d}{d t} \int_{0}^{\infty} \frac{\left(\Lambda^{-\alpha} u\right)(t, x)}{x^{\delta}} d x & =\int_{0}^{\infty} \frac{\Lambda^{-\alpha}\left(\Lambda^{-\alpha} u \partial_{x} u\right)}{x^{\delta}} d x-\nu \int_{0}^{\infty} \frac{\Lambda^{\beta} \Lambda^{-\alpha} u}{x^{\delta}} d x \\
& \geq C_{\alpha, \delta} \int_{0}^{\infty} \frac{\left(\Lambda^{-\alpha} u\right)^{2}}{x^{1+\delta}} d x-\nu C_{\beta, \delta} \int_{0}^{\infty} \frac{\Lambda^{-\alpha} u}{x^{\delta+\beta}} d x
\end{aligned}
$$

By Cauchy-Schwartz, we obtain

$$
\begin{aligned}
& \int_{0}^{\infty} \frac{\Lambda^{-\alpha} u}{x^{\delta+\beta}} d x \\
= & \int_{0}^{1} \frac{\Lambda^{-\alpha} u}{x^{\delta+\beta}} d x+\int_{1}^{\infty} \frac{\Lambda^{-\alpha} u}{x^{\delta+\beta}} d x \\
\leq & \left(\int_{0}^{1} \frac{\left(\Lambda^{-\alpha} u\right)^{2}}{x^{1+\delta}} d x\right)^{\frac{1}{2}}\left(\int_{0}^{1} \frac{1}{x^{\delta+2 \beta-1}} d x\right)^{\frac{1}{2}} \\
& \quad+\|u(t)\|_{L_{x}^{1}} \cdot\left\|\Lambda^{-\alpha}\left(|x|^{-\delta-\beta} \operatorname{sgn}(x) \chi_{|x| \geq 1}\right)\right\|_{L_{x}^{\infty} .}
\end{aligned}
$$


Observe that $\delta+2 \beta-1<1$ and $\delta+\beta>\alpha$. By Lemma 3.2 and Lemma 4.1, we get

$$
\int_{0}^{\infty} \frac{\Lambda^{-\alpha} u}{x^{\delta+\beta}} d x \leq \frac{C_{\alpha, \delta}}{2(1+\nu)} \int_{0}^{1} \frac{\left(\Lambda^{-\alpha} u\right)^{2}}{x^{1+\delta}} d x+C_{\alpha, \delta, \beta}\left(1+\left\|u_{0}\right\|_{L_{x}^{1}}\right) .
$$

Substituting this last estimate into (4.4) and again using Lemma 3.2 and CauchySchwartz, we obtain

$$
\begin{aligned}
\frac{d}{d t} \int_{0}^{\infty} \frac{\left(\Lambda^{-\alpha} u\right)(t, x)}{x^{\delta}} d x & \geq \frac{C_{\alpha, \delta}}{2} \int_{0}^{1} \frac{\left(\Lambda^{-\alpha} u\right)^{2}}{x^{1+\delta}} d x-C_{\alpha, \delta, \beta, \nu}\left(1+\left\|u_{0}\right\|_{L_{x}^{1}}\right) \\
& \geq C_{\alpha, \delta}^{\prime}\left(\int_{0}^{1} \frac{\left(\Lambda^{-\alpha} u\right)(t, x)}{x^{\delta}} d x\right)^{2}-C_{\alpha, \delta, \beta, \nu}\left(1+\left\|u_{0}\right\|_{L_{x}^{1}}\right) \\
& \geq C_{\alpha, \delta}^{\prime \prime}\left(\int_{0}^{\infty} \frac{\left(\Lambda^{-\alpha} u\right)(t, x)}{x^{\delta}} d x\right)^{2}-C_{\alpha, \delta, \beta, \nu}^{\prime}\left(1+\left\|u_{0}\right\|_{L_{x}^{1}}\right)^{2} .
\end{aligned}
$$

It is now clear that if $u_{0}$ satisfy (1.2) with sufficiently large constant $C_{\alpha, \beta, \delta, \nu}$, then we obtain the inequality of the form

$$
\frac{d}{d t} a(t) \geq C \cdot a(t)^{2}
$$

where

$$
a(t)=\int_{0}^{\infty} \frac{\left(\Lambda^{-\alpha} u\right)(t, x)}{x^{\delta}} d x
$$

Clearly $a(t)$ goes to infinity in finite time. We have obtained the desired contradiction and the proof is now completed.

\section{ACKNOWLEDGEMEnTS}

The first author was partially supported by the NSF under agreements DMS0800129 and DMS-1056737. The second author was supported in part by the NSF under agreement No. DMS-1128155. Any opinions, findings and conclusions or recommendations expressed in this material are those of the authors and do not necessarily reflect the views of the National Science Foundation. The second author was also supported in part by an NSerc discovery grant.

\section{REFERENCES}

[1] Nathaël Alibaud, Jérôme Droniou, and Julien Vovelle, Occurrence and non-appearance of shocks in fractal Burgers equations, J. Hyperbolic Differ. Equ. 4 (2007), no. 3, 479-499, DOI 10.1142/S0219891607001227. MR2339805 (2008i:35198)

[2] Piotr Biler, Tadahisa Funaki, and Wojbor A. Woyczynski, Fractal Burgers equations, J. Differential Equations 148 (1998), no. 1, 9-46, DOI 10.1006/jdeq.1998.3458. MR1637513 (99g:35111)

[3] Angel Castro, Diego Córdoba, and Francisco Gancedo, Singularity formations for a surface wave model, Nonlinearity 23 (2010), no. 11, 2835-2847, DOI 10.1088/0951-7715/23/11/006. MR2727172(2011k:35198)

[4] Dongho Chae, Peter Constantin, and Jiahong Wu, Inviscid models generalizing the twodimensional Euler and the surface quasi-geostrophic equations, Arch. Ration. Mech. Anal. 202 (2011), no. 1, 35-62, DOI 10.1007/s00205-011-0411-5. MR 2835862 (2012j:76017)

[5] Chi Hin Chan and Magdalena Czubak, Regularity of solutions for the critical $N$-dimensional Burgers' equation, Ann. Inst. H. Poincaré Anal. Non Linéaire 27 (2010), no. 2, 471-501, DOI 10.1016/j.anihpc.2009.11.008 (English, with English and French summaries). MR2595188 (2011e:35332) 
[6] Antonio Córdoba and Diego Córdoba, A maximum principle applied to quasi-geostrophic equations, Comm. Math. Phys. 249 (2004), no. 3, 511-528, DOI 10.1007/s00220-004-1055-1. MR2084005 (2005f:76011)

[7] Antonio Córdoba, Diego Córdoba, and Marco A. Fontelos, Formation of singularities for a transport equation with nonlocal velocity, Ann. of Math. (2) 162 (2005), no. 3, 1377-1389, DOI 10.4007/annals.2005.162.1377. MR2179734(2007b:35011)

[8] Antonio Córdoba, Diego Córdoba, and Marco A. Fontelos, Integral inequalities for the Hilbert transform applied to a nonlocal transport equation, J. Math. Pures Appl. (9) 86 (2006), no. 6, 529-540, DOI 10.1016/j.matpur.2006.08.002 (English, with English and French summaries). MR2281451(2007k:35040)

[9] Diego Córdoba, Marco A. Fontelos, Ana M. Mancho, and Jose L. Rodrigo, Evidence of singularities for a family of contour dynamics equations, Proc. Natl. Acad. Sci. USA 102 (2005), no. 17, 5949-5952, DOI 10.1073/pnas.0501977102. MR2141918 (2006j:76015)

[10] Hongjie Dong, Well-posedness for a transport equation with nonlocal velocity, J. Funct. Anal. 255 (2008), no. 11, 3070-3097, DOI 10.1016/j.jfa.2008.08.005. MR2464570 (2010b:35056)

[11] Hongjie Dong and Dapeng Du, Global well-posedness and a decay estimate for the critical dissipative quasi-geostrophic equation in the whole space, Discrete Contin. Dyn. Syst. 21 (2008), no. 4, 1095-1101, DOI 10.3934/dcds.2008.21.1095. MR2399451(2009b:76175)

[12] Hongjie Dong and Dong Li, Finite time singularities for a class of generalized surface quasi-geostrophic equations, Proc. Amer. Math. Soc. 136 (2008), no. 7, 2555-2563, DOI 10.1090/S0002-9939-08-09328-3. MR2390526 (2009c:35361)

[13] Hongjie Dong, Dapeng Du, and Dong Li, Finite time singularities and global well-posedness for fractal Burgers equations, Indiana Univ. Math. J. 58 (2009), no. 2, 807-821, DOI 10.1512/iumj.2009.58.3505. MR2514389(2010i:35320)

[14] Francisco Gancedo, Existence for the $\alpha$-patch model and the $Q G$ sharp front in Sobolev spaces, Adv. Math. 217 (2008), no. 6, 2569-2598, DOI 10.1016/j.aim.2007.10.010. MR2397460 (2009b:35329)

[15] A. Kiselev, F. Nazarov, and A. Volberg, Global well-posedness for the critical 2D dissipative quasi-geostrophic equation, Invent. Math. 167 (2007), no. 3, 445-453, DOI 10.1007/s00222006-0020-3. MR2276260(2008f:35308)

[16] Alexander Kiselev, Fedor Nazarov, and Roman Shterenberg, Blow up and regularity for fractal Burgers equation, Dyn. Partial Differ. Equ. 5 (2008), no. 3, 211-240. MR2455893 (2009k:35264)

[17] Alexander Kiselev, Nonlocal maximum principles for active scalars, Adv. Math. 227 (2011), no. 5, 1806-1826, DOI 10.1016/j.aim.2011.03.019. MR.2803787 (2012k:35414)

[18] Dong Li and Jose Rodrigo, Blow-up of solutions for a $1 D$ transport equation with nonlocal velocity and supercritical dissipation, Adv. Math. 217 (2008), no. 6, 2563-2568, DOI 10.1016/j.aim.2007.11.002. MR2397459(2009c:35029)

[19] Dong Li and Jose Rodrigo, Blow up for the generalized surface quasi-geostrophic equation with supercritical dissipation, Comm. Math. Phys. 286 (2009), no. 1, 111-124, DOI 10.1007/s00220-008-0585-3. MR2470925 (2010a:86014)

[20] Changxing Miao and Gang Wu, Global well-posedness of the critical Burgers equation in critical Besov spaces, J. Differential Equations 247 (2009), no. 6, 1673-1693, DOI 10.1016/j.jde.2009.03.028. MR 2553854 (2010j:35468)

[21] Hisashi Okamoto, Takashi Sakajo, and Marcus Wunsch, On a generalization of the Constantin-Lax-Majda equation, Nonlinearity 21 (2008), no. 10, 2447-2461, DOI 10.1088/0951-7715/21/10/013. MR2439488 (2009g:76009)

Division of Applied Mathematics, Brown University, 182 George Street, Providence, RHODE ISLAND 02912

E-mail address: Hongjie_Dong@brown.edu

Department of Mathematics, University of British Columbia, Vancouver, British Columbia, Canada V6T $1 \mathrm{Z2}$

E-mail address: mpdongli@gmail.com 